\title{
Synthesis and nano-mechanical properties of polystyrene/silica core/shell particles via atomic force microscopy
}

\author{
Hamid Akbari Moayyer a,b, Malek Naderi a,b* , Jamshid Aghazadeh Mohandesi ${ }^{\mathrm{b}}$ \\ ${ }^{a}$ Graphene and Advanced Materials Laboratory (GAMLab), Amirkabir University of Technology, Tehran, \\ Iran \\ ${ }^{\mathrm{b}}$ Department of Materials and Metallurgical Engineering, Amirkabir University of Technology, Tehran, Iran
}

Corresponding author: mnaderi@aut.ac.ir

\section{Data process}

The contact radius cannot be acquired directly in AFM experiments. Therefore, the indentation depth $h$ was measured and used to calculate the elastic modulus. The force-displacement diagrams must be converted into force-indentation curves to calculate the elastic modulus ${ }^{1-2}$. The applied load $F$ between a cantilever and a sample can be calculated as follows:

$F=k d$

where $k$ and $d$ are the spring constant and deformation of the cantilever. The indentation depth $h$ of the tip into the sample surface is:

$h=z-d$

in which $z$ is the piezo displacement. Thermal drifts in the components and stresses in the cantilever cause the height offset $z_{0}$ and the deflection offset $d_{0}{ }^{2}$. By taking these into account, Equations (S1) and (S2) could be rewritten as follows:

$F=k\left(d-d_{0}\right)$

$h=\left(z-z_{0}\right)-\left(d-d_{0}\right)$

Fig. S1 shows typical force-indentation curves for PS and $\mathrm{PS} / \mathrm{SiO}_{2}$ particles. The results are obtained using a DLC probe. Also, curves were fitted with the Hertz model (dashed line). 


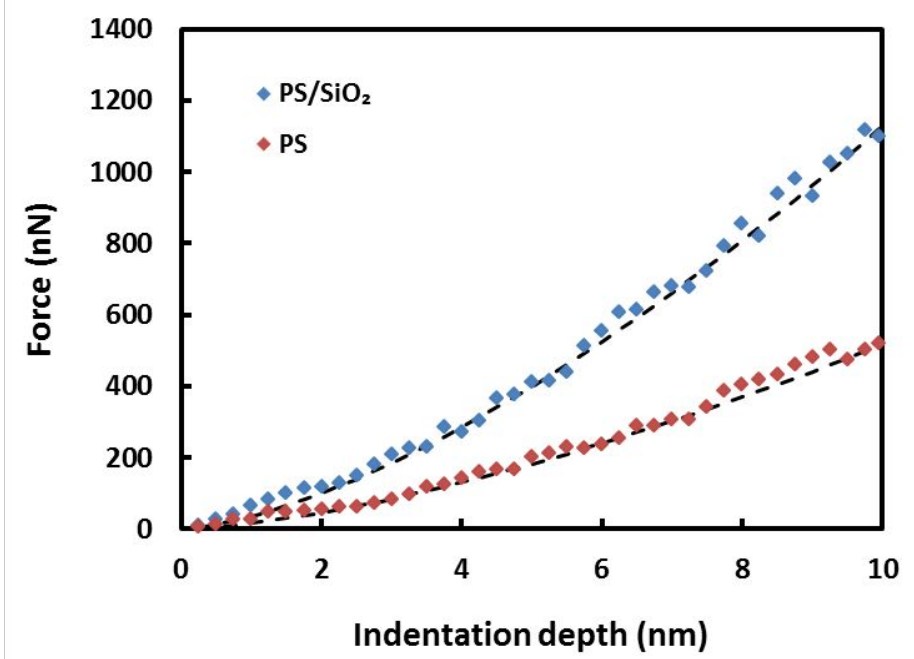

Fig. S1. Typical force-indentation curves for PS and $\mathrm{PS} / \mathrm{SiO}_{2}$ particles through DLC probe. The curves were fitted with the Hertz model (dashed line).

\section{Models of contact mechanics}

To extract the elastic modulus from the force-indentation depth curves, it is necessary to use models of contact mechanics. Numerous contact models have been developed including the Hertz ${ }^{3}$, JKR ${ }^{4}$, and DMT ${ }^{5}$ models. In the Hertz theory, adhesion forces between two surfaces are ignored, while in the JKR and DMT models, adhesion forces inside and outside the contact area are considered in the calculations, respectively ${ }^{6}$. Recent studies in the nanoindentation of polystyrene and silica particles using Si and DLC probes have revealed the JKR model fits better than the DMT in these systems ${ }^{1,7}$. Therefore, Hertz and JKR contact models were used in the current study.

The Hertz theory describes the contact of two surfaces in the simplest way, which is the most used approach ${ }^{3}$. This model does not take the adhesion force into account and is valid for nonadhesive elastic spheres with small deformations ${ }^{8}$. As two elastic spheres come in contact with each other with an applied normal load $F$, a circular contact zone of radius $a$ formed at their interface that can be expressed as 3,8 :

$a=\left(\frac{3 R^{*} F}{4 E^{*}}\right)^{1 / 3}$

in which $R^{*}$ is the relative radius of curvature and $E^{*}$ is the combined modulus, which are defined as follows:

$\frac{1}{R^{*}}=\frac{1}{R_{1}}+\frac{1}{R_{2}}$

$\frac{1}{E^{*}}=\frac{1-\vartheta_{1}^{2}}{E_{1}}+\frac{1-\vartheta_{2}^{2}}{E_{2}}$

where $R, v$, and $E$ are the radius of curvature, Poisson's ratio, and elastic modulus, respectively. The subscript 1 indicates the tip and 2 represents the particle. The deformation of the particle $h$ can be expressed as:

$h=\frac{a^{2}}{R^{*}}=\left(\frac{9 F^{2}}{16 R^{*} E^{* 2}}\right)^{1 / 3}$ 
According to Equation (S8), the combined modulus and elastic modulus of the particle can be obtained as:

$$
\begin{aligned}
& E^{*}=\frac{3}{4} \times \frac{F}{h^{3 / 2} R^{* 1 / 2}} \\
& E_{2}=\left(1-\vartheta_{2}^{2}\right) \times\left(\frac{1}{E^{*}}-\frac{1-\vartheta_{1}^{2}}{E_{1}}\right)^{-1}
\end{aligned}
$$

The Johnson-Kendall-Roberts (JKR) model considers adhesion forces inside the contact zone. This model can be applied to highly adhesive materials with low stiffness ${ }^{8}$. In this model, the work of adhesion $W$ and the load between the tip and the particle $F_{J K R}$ are defined as ${ }^{1}$ :

$W=\frac{2 F_{a d}}{3 \pi R^{*}}$

$$
F_{J K R}=F+3 \pi W R^{*}+\sqrt{6 \pi W R^{*} F+9 \pi^{2} W^{2} R^{* 2}}
$$

where $F_{a d}$ is the adhesion force between the tip and the particle, which can be extracted from the retract curve of the force-displacement diagram. The radius of the contact region $a_{J K R}$ and the indentation depth $h_{J K R}$ are expressed as:

$$
\begin{aligned}
& a_{J K R}=\left(\frac{3 R^{*} F_{J K R}}{4 E^{*}}\right)^{1 / 3} \\
& h_{J K R}=\frac{a_{J K R}^{2}}{R^{*}}-\sqrt{\frac{2 \pi W a_{J K R}}{E^{*}}}
\end{aligned}
$$

The combined modulus and elastic modulus of a particle can be obtained using Equation (S11) to (S14):

$$
\begin{aligned}
& E^{*}=\left(\frac{\left(\frac{3}{4}\right)^{2 / 3} F_{J K R}^{2 / 3}-\left(\frac{3}{4}\right)^{1 / 6}\left(2 \pi R^{*} W\right)^{1 / 2} F_{J K R}^{1 / 6}}{R^{*}{ }^{1 / 3} h_{J K R}}\right)^{3 / 2} \\
& E_{2}=\left(1-\vartheta_{2}^{2}\right) \times\left(\frac{1}{E^{*}}-\frac{1-\vartheta_{1}{ }^{2}}{E_{1}}\right)^{-1}
\end{aligned}
$$




\section{References}

1. Huang, P.; Zhang, L.; Yan, Q.; Guo, D.; Xie, G., Size Dependent Mechanical Properties of Monolayer Densely Arranged Polystyrene Nanospheres. Langmuir : the ACS journal of surfaces and colloids 2016, 32 (49), 13187-13192.

2. Guo, D.; Li, J.; Xie, G.; Wang, Y.; Luo, J., Elastic properties of polystyrene nanospheres evaluated with atomic force microscopy: size effect and error analysis. Langmuir : the ACS journal of surfaces and colloids 2014, 30, 7206-12.

3. Hertz, H.; Reine, J., On the contact of elastic solids. Angew. Math. 1882, 92, 156-171.

4. Johnson, K. L.; Kendall, K.; Roberts, A. D. In Surface energy and the contact of elastic solids, Proc. R. Soc. London A, 1971; p 301-313.

5. Derjaguin, B. V.; Muller, V. M.; Toporov, Y. P., Effect of contact deformations on the adhesion of particles. J. Coll. Interf. Sci. 1975, 53, 314-326.

6. Butt, H. J.; Cappella, B.; Kappl, M., Force measurements with the atomic force microscope: Technique, interpretation and applications. Surf. Sci. Rep. 2005, 59, 1-152.

7. Cao, X.; Pan, G.; Huang, P.; Guo, D.; Xie, G., Silica-Coated Core-Shell Structured Polystyrene Nanospheres and Their Size-Dependent Mechanical Properties. Langmuir : the ACS journal of surfaces and colloids 2017, 33 (33), 8225-8232.

8. Johnson, K. L., Contact mechanics. Cambridge University Press: Cambridge, U.K., 1985. 